\title{
A New Cascaded Framework for Lithium-Ion Battery State and Parameter Estimation
}

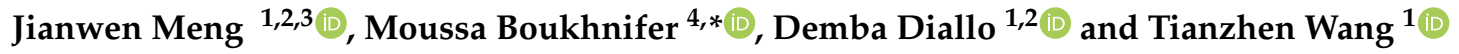 \\ 1 Department of Electrical Automation, Shanghai Maritime University, Shanghai 201306, China; \\ Jianwen.meng@estaca.fr (J.M.); demba.diallo@geeps.centralesupelec.fr (D.D.); tzwang@shmtu.edu.cn (T.W.) \\ 2 Laboratoire de Génie Electrique et Electronique de Paris, CNRS, CentraleSupélec, Université Paris-Saclay, \\ 91192 Gif-sur-Yvette, France \\ 3 ESTACA, Ecole d'Ingénieurs, 12 avenue Paul Delouvrier, 78066 Saint-Quentin-en-Yvelines, France \\ 4 LCOMS, Université de Lorraine, 57000 Metz, France \\ * Correspondence: moussa.boukhnifer@univ-lorraine.fr
}

Received: 19 December 2019; Accepted: 23 January 2020; Published: 4 February 2020

\begin{abstract}
Lithium-ion battery on-line monitoring is challenging due to the unmeasurable characteristic of its internal states. Up to now, the most effective approach for battery monitoring is to apply advanced estimation algorithms based on equivalent circuit models. Besides, a usual method for estimating slowly varying unmeasurable parameters is to include them in the state vector with the zero-time derivative condition, which constitutes the so-called extended equivalent circuit model and has been widely used for the battery state and parameter estimation. Although various advanced estimation algorithms are applied to the joint estimation and dual estimation frameworks, the essence of these estimation frameworks has not been changed. Thus, the improvement of the battery monitoring result is limited. Therefore, a new battery monitoring structure is proposed in this paper. Firstly, thanks to the superposition principle, two sub-models are extracted. For the nonlinear one, an observability analysis is conducted. It shows that the necessary conditions for local observability depend on the battery current, the initial value of the battery capacity, and the square of the derivative of the open circuit voltage with respect to the state of charge. Then, the obtained observability analysis result becomes an important theoretical support to propose a new monitoring structure. Commonly used estimation algorithms, namely the Kalman filter, extended Kalman filter, and unscented Kalman filter, are selected and employed for it. Apart from providing a simultaneous estimation of battery open circuit voltage, more rapid and less fluctuating battery capacity estimation are the main advantages of the new proposed monitoring structure. Numerical studies using synthetic data have proven the effectiveness of the proposed framework.
\end{abstract}

Keywords: lithium-ion battery; battery monitoring; extended equivalent circuit model; observability analysis; Kalman filtering

\section{Introduction}

As a typical type of electrochemical power source, lithium-ion batteries (LIBs) undergo degradation in both energy capacity and internal resistance during their irreversible aging process [1]. Hence, a reliable and efficient operation of LIBs requires monitoring, control, and management [2,3]. A battery management system (BMS) is of great importance for the batteries, and state estimation is one of its key roles [4]. Accurately assessing the battery state of charge (SOC) and state of health $(\mathrm{SOH})$ can not only make batteries work closer to their physical limits, but also guarantee their safety and lifetime [5]. However, due to the unmeasurable characteristic of these indicators, battery monitoring is challenging. With a mathematical model that characterizes the dynamics of the battery, indicators 
such as SOC, $\mathrm{SOH}$, etc., need to be inferred from the limited available measurements such as current, terminal voltage, and surface temperature [6].

Up to now, the most effective approach for battery monitoring is to apply advanced estimation algorithms based on equivalent circuit models (ECMs) [7]. The ECM composed of an open circuit voltage (OCV) source connected in series with a resistor and one or more RC networks is widely used to reproduce the battery's electrical behavior and to further accomplish the objective of battery state estimation [8]. However, the model parameters are actually affected by many factors such as temperature, SOC, charge/discharge rates, and battery aging [4]. Hence, the state-of-the-art estimation methods usually focus on an on-line identified battery model, namely the adaption of model parameters is indispensable. In [9], the parameters of a Thevenin model were constantly updated by a forgetting factor recursive least squares algorithm, while the nonlinear Kalman filter was used to perform the SOC estimation. The series combination of the commonly used recursive least squares (RLS) method and nonlinear state estimator provides a feasible way to enhance the estimation accuracy. In addition, a multi-timescale estimator with a vector-type RLS parameter adaption mechanism was proposed in [4]. The vector-type RLS can address the different variation rates of battery parameters, and then, Kalman filter-based SOC estimator and RLS-based capacity estimator are formulated and integrated in the form of dual estimation. Furthermore, considering the the current and voltage sensors are usually corrupted by noises, a novel technique that integrates the Frisch scheme-based bias compensating RLS with a SOC observer was proposed in [10]. The proposed method estimates the noise statistics and compensates the noise effect so that the model parameters can be extracted without bias. Similarly, the on-line model parameterization process considering the measurement noises was discussed in [11,12] recently.

In fact, the aforementioned battery monitoring process can be eventually converted to a classical state and parameter estimation problem. A usual method for estimating slowly varying unmeasurable parameters is to include them in the state vector with the zero-time derivative condition [13]. We can obtain the so-called battery extended ECM. Then, joint or dual estimation methods, mainly based on single or double observers, are preferred [14] and can be applied to the battery extended ECM. Apart from enhancing the accuracy of state estimation, the estimated parameters can also be used to build a battery $\mathrm{SOH}$ indicator. $\mathrm{SOH}$, usually evaluated by some related battery parameters correlated with their aging degrees, can be effectively estimated with the zero-time derivative condition. SOC, as the battery energy state indicator, usually appears as a state variable derived from the common ampere-hour integral method [15].

On the one hand, the joint estimation method based on a single observer can cope with the battery monitoring problem in high-dimensional state space directly. For instance, a single extended Kalman filter (EKF) was applied to the joint estimation structure for battery state and parameter estimation in [16]. Improvement of the battery monitoring performance with the joint estimation method using the unscented Kalman filter (UKF) can be found in [17]. Furthermore, simultaneous battery OCV estimation is realized with adaptive EKF in the joint estimation framework in [18]. Recently, considering the model uncertainties in [19], H $\infty$ EKF was introduced to the joint estimation structure in order to have a robust estimation of battery SOC and ohmic and polarization resistance simultaneously.

On the other hand, a more flexible estimation framework based on two observers is called the dual estimation method. The double EKF [14], double UKF [17], double sliding mode observer [20], double $\mathrm{H} \infty$ observer [21], and double particle filter [22] are introduced to the dual estimation framework, where battery parameters and states can be estimated separately with different time scales. Additionally, merging the advantages of different filtering techniques is another objective of the dual estimation method. For example, the UKF and $\mathrm{H} \infty$ observer were applied in the dual estimation framework in [23], in which the $\mathrm{H} \infty$ observer was used to have a robust battery parameter estimation, and more accurate SOC estimation was achieved with UKF.

Although fruitful research works have been brought based on the two aforementioned classical structures, the essence of the different observer-based battery monitoring studies has not changed. The main existing problem in the current two monitoring structures will be intuitively pointed out 
by a representative research work as follows. In [24], the multi-time scale dual estimation framework was studied with EKF. As shown in Figure 1, an interesting phenomenon can be observed from the experimental results. It raises several questions.

1. Why is the convergence speed of the battery usable capacity so slow?

A similar problem can also be found in other research works such as [2,14], etc. Although they explained that the capacity estimation is faster than the capacity degradation, the slow convergence speed of battery usable capacity in classical monitoring structures, usually several hours, still needs to be explored.

2. Why will the smaller initial value of battery usable capacity lead to a rapid convergence speed?

As can be observed from Figure 1a-d, although the steady state error was similar for 1 Ah or 2 Ah, the convergence speed of the estimation was faster with the lowest initial value.

3. Are there other observability conditions that can guarantee a rapid convergence speed for the battery usable capacity?

Based on the above question, how to find the other observability conditions to improve the battery monitoring result is of great importance. Indeed, the convergence rate can be adjusted by varying the process and measurement noise covariance during the observer design process [24]. However, intrinsic characteristics from the battery or the used model should be mastered, whether we can take advantage of them or effectively avoid them.

4. How can we improve the battery monitoring performance based on the classical extended ECM, if the answers to the aforementioned questions are found?

That is, how can we take advantage of the existing literature, including the validated model and experimental information, to propose a new battery monitoring structure that can overcome the drawbacks of the traditional joint and dual estimation frameworks?
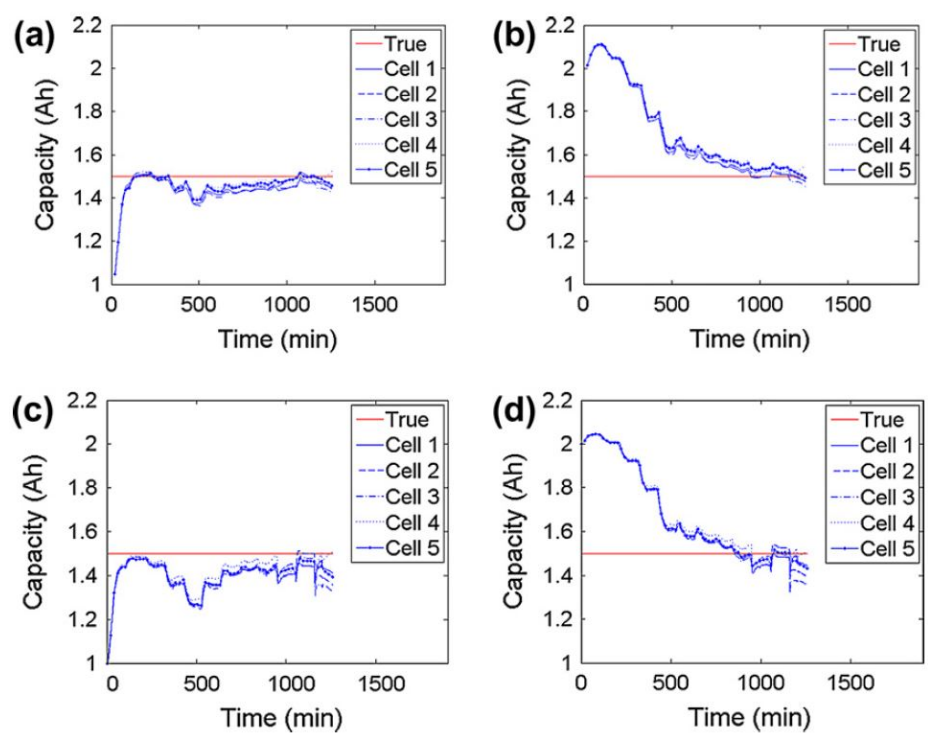

Figure 1. Battery usable capacity estimation result with the dual estimation framework [24], Copyright Elsevier, 2012. (a,b) are the capacity estimation result with a multi-time scale; (c,d) are the capacity estimation result with a single time scale. Both of these two experimental validations are tested with smaller (1 Ah) and larger (2 Ah) initial values in EKF than the true value of $1.5 \mathrm{Ah}$ (Ah: ampere-hour).

Hence, the contribution of this paper lies in answering the four previously proposed questions. Namely: 
- Thanks to the superposition principle, two sub-models are extracted. For the nonlinear one, an observability analysis is conducted. The local observability for battery ECM parameters and battery usable capacity are analyzed separately.

- The necessary observability conditions for the extended ECM are derived and clearly listed. It shows that the necessary conditions for local observability depend on the battery current value, the initial value of the battery capacity, and the square of the derivative of the OCV with respect to the SOC.

- A new cascaded framework for the LIB state and parameter estimation is proposed based on the obtained theoretical analysis results. The battery ECM parameter estimation and battery capacity estimation are divided into two parts. A simultaneous estimation of OCV will connect these two parts.

- The derived local observability conditions and the new proposed framework extend the traditional battery monitoring study.

The remainder of this paper is organized as follows. Firstly, details of the extended battery ECM will be introduced in Section 2. Secondly, a theoretic and systematic observability analysis for the nonlinear extended battery ECM will be developed in Section 3, where the necessary local observability conditions of the battery state and parameter estimation will be clearly listed. Thirdly, a new cascaded framework for battery monitoring, with a main improvement for the battery usable capacity estimation, will be proposed in Section 4. Furthermore, Section 5 is dedicated to the numerical evaluation of the estimation framework. Last, but not least, conclusions will be drawn in Section 6.

\section{Battery ECM and Extended Battery ECM}

\subsection{Battery ECM}

The considered battery ECM is shown in Figure 2. It is one of the most widely used ECMs [6], and it includes a double RC network, which is a good trade-off between the error and the complexity of the model compared with single and triple RC structures [25].

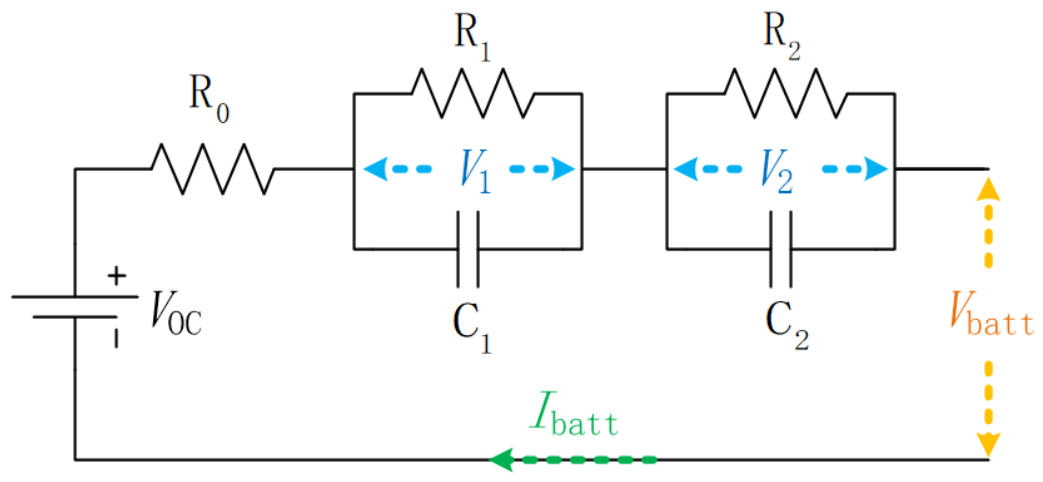

Figure 2. Battery equivalent circuit model (ECM) with the double RC network.

The resistor $\mathrm{R}_{0}$ stands for ohmic resistance, which includes the resistance of contacts, electrodes, as well as electrolytes [26]. The double pair RC captures the transient battery dynamics such as the charge transfer kinetics, the lithium ion diffusion, and solid/electrolyte interface (SEI) dynamics [8].

The voltage source $V_{\mathrm{OC}}$ represents the OCV, which mainly depends on the battery SOC [8]. As an example shown in Figure 3, its average value, $V_{\mathrm{OC}}(s o c)$, is usually a monotonically increasing function of SOC [27]. In each SOC interval, it can be approximated by $V_{\mathrm{OC} i}(\operatorname{soc})=a_{i} \cdot \operatorname{soc}+b_{i}\left(a_{i}\right.$ and $b_{i}$ are constant in the $i$ th SOC interval). The nonlinearity of this curve is inevitable. 


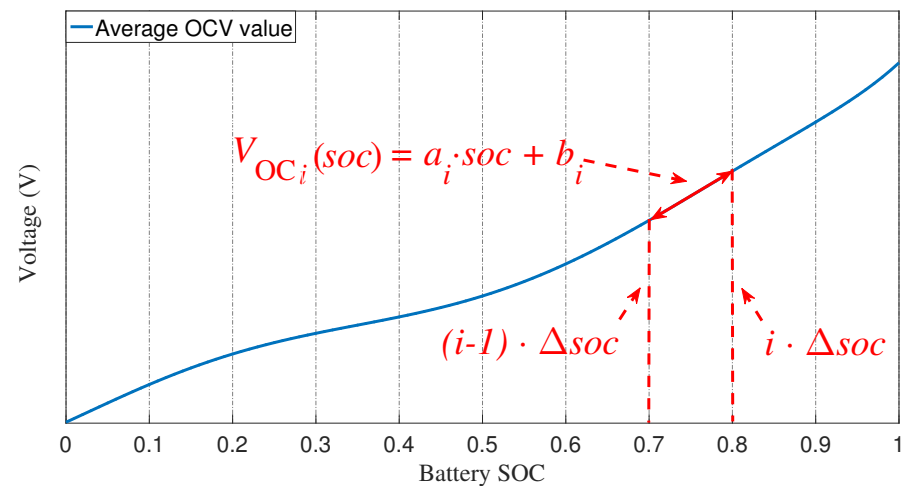

Figure 3. General form of the average open circuit voltage (OCV) curve (example adapted from [28], Copyright IEEE, 2013).

Furthermore, the battery SOC $\in[0 \%, 100 \%]$ can be modeled by the classical Coulomb counting method [28]:

$$
\operatorname{soc}(t)=\operatorname{soc}\left(t_{0}\right)-\eta \int_{t_{0}}^{t} \frac{I_{\text {batt }}(\tau)}{C_{\mathrm{n}}} d \tau
$$

where soc is the operator of SOC; $\eta$ is the Coulombic efficiency; $C_{n}(A h)$ is the battery usable capacity; $\operatorname{soc}(t)$ is the required SOC at time point $t$ based on its initial value $\operatorname{soc}\left(t_{0}\right)$.

Therefore, the battery dynamic behavior can be described by the following state-space representation:

$$
\begin{aligned}
& {\left[\begin{array}{c}
\frac{\mathrm{d} V_{1}(t)}{\mathrm{d} t} \\
\frac{\mathrm{d} V_{2}(t)}{\mathrm{d} t} \\
\frac{\mathrm{d} s o c(t)}{\mathrm{d} t}
\end{array}\right]=M\left[\begin{array}{c}
V_{1}(t) \\
V_{2}(t) \\
s o c(t)
\end{array}\right]+N I_{\text {batt }}(t)} \\
& V_{\text {batt }}(t)=V_{\mathrm{OC}}(s o c)-V_{1}(t)-V_{2}(t)-\mathrm{R}_{0} I_{\text {batt }}(t)
\end{aligned}
$$

where,

$$
\boldsymbol{M}=\left[\begin{array}{ccc}
\frac{-1}{\mathrm{R}_{1} \mathrm{C}_{1}} & 0 & 0 \\
0 & \frac{-1}{\mathrm{R}_{2} \mathrm{C}_{2}} & 0 \\
0 & 0 & 0
\end{array}\right], \quad \boldsymbol{N}=\left[\begin{array}{c}
\frac{1}{\mathrm{C}_{1}} \\
\frac{1}{\mathrm{C}_{2}} \\
\frac{-\eta}{\mathrm{C}_{\mathrm{n}}}
\end{array}\right]
$$

and $V_{\text {batt }}$ is the battery output voltage. $V_{1}$ and $V_{2}$ are the voltages across the capacitors $C_{1}$ and $C_{2}$, respectively; $I_{\text {batt }}$ is the battery input current, according to its reference direction in Figure 2 ; " + " means discharging process, while "-" means charging process.

\subsection{Extended Battery ECM}

To take into account the parametric variation due to the aging process, the relationship $\frac{\mathrm{d} P}{\mathrm{~d} t} \approx 0$ will be considered to build an extended battery ECM, where $P$ is a general representation of the parameters such as $R_{0}, R_{1}, R_{2}, C_{1}, C_{2}$, and $C_{n}$. Details for the zero-time derivative condition for battery parameters can be found in [29]. Hence, the extended battery model is given in (3).

It has been shown that the parameter variation of the second $\mathrm{RC}$ network during the aging process can be neglected [19]. Therefore, $R_{2}$ and $C_{2}$ are considered as constant. Hence, only the four parameters $R_{0}, R_{1}, C_{1}$, and $C_{n}$ are considered as additional state variables and will be estimated with the SOC. 
The updating of these variables will cancel or at least mitigate the battery aging effect or temperature variation on the monitoring [23]. Finally, the extended battery model is as follows:

$$
\begin{aligned}
& \left\{\begin{aligned}
\frac{\mathrm{d} V_{1}(t)}{\mathrm{d} t} & =\frac{-1}{\mathrm{R}_{1}(t) \mathrm{C}_{1}(t)} V_{1}(t)+\frac{1}{\mathrm{C}_{1}(t)} I_{\text {batt }}(t) \\
\frac{\mathrm{d} V_{2}(t)}{\mathrm{d} t} & =\frac{-1}{\mathrm{R}_{2} C_{2}} V_{2}(t)+\frac{1}{\mathrm{C}_{2}} I_{\text {batt }}(t) \\
\frac{\mathrm{d} s o c(t)}{\mathrm{d} t} & =\frac{-\eta I_{\text {batt }}(t)}{\mathrm{C}_{\mathrm{n}}(t)} \\
\frac{\mathrm{d} \mathrm{R}_{0}(t)}{\mathrm{d} t} & \approx 0 \\
\frac{\mathrm{dR}}{\mathrm{d} t}(t) & \approx 0 \\
\frac{\mathrm{d} C_{1}(t)}{\mathrm{d} t} & \approx 0 \\
\frac{\mathrm{d} C_{\mathrm{n}}(t)}{\mathrm{d} t} & \approx 0
\end{aligned}\right. \\
& V_{\text {batt }}(t)=V_{\mathrm{OC}}(\operatorname{soc})-V_{1}(t)-V_{2}(t)-\mathrm{R}_{0}(t) \cdot I_{\text {batt }}(t)
\end{aligned}
$$

\section{Local Observability Conditions for the Extended Battery ECM}

The concept of observability is useful in reconstructing the unmeasurable state variables from the measurable signals. Therefore, assessing the observability for the used model before executing the state and parameter estimation is necessary. However, due to the numerous states and nonlinearity, observability analysis for the extended battery model (3) is challenging and usually overlooked by most of the research works.

In [6], linear and nonlinear observability analysis methods were compared for the battery SOC estimation with ECM. It was pointed out that the local observability of a nonlinear dynamic system at an operating point is not the same as the observability of the system linearized around this point. Recently, the observability analysis for the extended ECM without extending the battery capacity was done in [19], where non-zero input battery current was deduced as the observability condition. However, the observability analysis for the extended battery model (3) needs further discussion.

Hence, a theoretic and systematic observability analysis method is proposed specifically for the extended battery ECM in this paper. Firstly, the characteristic of the battery ECM is used to decompose the original ECM into two sub-models. Then, nonlinear observability analysis method is applied for each sub-model. A schematic diagram of the proposed analysis method is shown in Figure 4, where the arrows represent the analysis process.

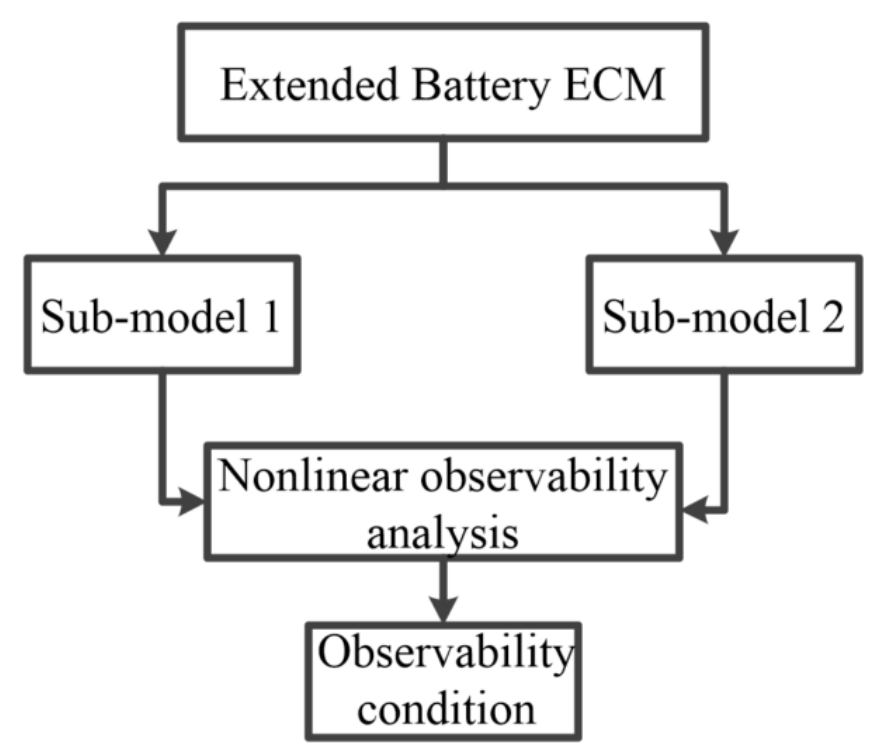

Figure 4. Schematic diagram of the analysis process. 


\subsection{Battery ECM Decomposition}

In essence, the nonlinearity of the battery model is due to $V_{\mathrm{OC}}$. Therefore, researchers generally resort to the linearized model to simplify the analysis process. The observability is studied in different SOC operating intervals as shown in Figure 3 [6].

Based on the same approach, we consider that in each SOC interval, the ECM is a linear model of the battery. Hence, the superposition principle can be applied [30]. The battery ECM displayed in Figure 5a is split into two sub-models:

- in Figure 5b, only the current source is considered;

- in Figure 5c, the current source is disconnected and only the voltage source is under consideration.

One sub-model has two states, namely $V_{1}$ and $V_{2}$; while the other one, which is only composed of the OCV voltage source, has only one state, which is SOC.

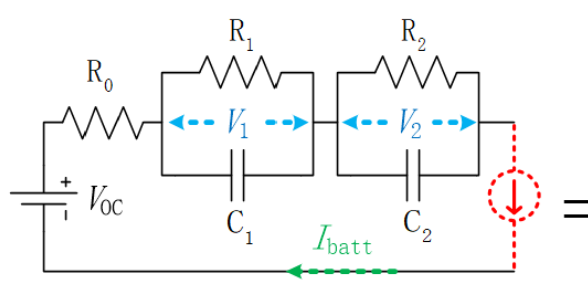

(a) Original model

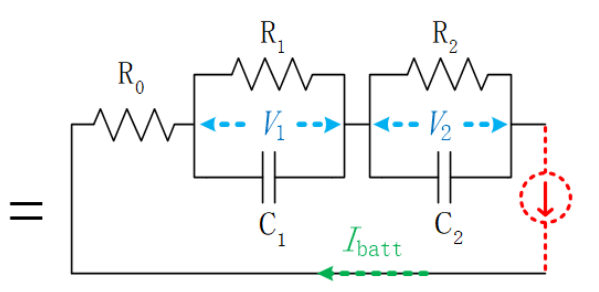

(b) Sub-model 1

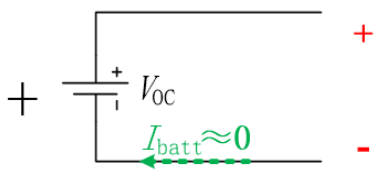

(c) Sub-model 2

Figure 5. Battery ECM decomposition according to the superposition principle.

\subsection{Observability Analysis for the Extended Sub-Models}

The observability for the first sub-model depicted in Figure 5b was already studied in [31]. The conclusion was that the time varying behavior of battery current $I_{\text {batt }}$ was of utmost importance to guarantee good observability conditions for the estimation of $R_{0}, R_{1}, C_{1}$. To be specific, in order to estimate the ECM parameters without affecting the battery discharging process, an additional pseudo random binary signal (PRBS) can be added to the battery constant charging current by the charging controller. The added PRBS provides a persistently exciting signal, which makes the extended system observable [31].

Therefore, only the observability analysis for the second sub-model will be developed in the following. When the current source is disconnected, $I_{\mathrm{batt}}=I_{\mathrm{d}}$, where $I_{\mathrm{d}}$ stands for the self-discharge current. This current is naturally very small compared to the current that flows in or out of the battery when connected to the load. Therefore, the voltage across $\mathrm{R}_{0}$ and the RC networks can be neglected when compared to $V_{\mathrm{OC}}$.

Eventually, the state-space representation of this sub-model can be written as:

$$
\begin{aligned}
& \left\{\begin{array}{l}
\frac{\mathrm{d} s o c(t)}{\mathrm{d} t}=\frac{-\eta I_{\text {batt }}(t)}{C_{\mathrm{n}}(t)} \\
\frac{\mathrm{d} C_{\mathrm{n}}(t)}{\mathrm{d} t} \approx 0
\end{array}\right. \\
& y_{2}=V_{\mathrm{OC}}(s o c)
\end{aligned}
$$

Besides, one can first recall that a numerical determination theorem for the local observability of a nonlinear model $\left\{\begin{array}{l}\dot{x}=f(x, u) \\ y=h(x, u)\end{array}\right.$, where $x$ is an $n \times 1$ state vector, $y$ is a scalar output, $u$ is a scalar system input, $f(\cdot)$ and $h(\cdot)$ are nonlinear functions, at point $x^{0}$ is as follows [31]:

Theorem 1. If the following Jacobian matrix $\mathbf{J}$ has full rank, then the nonlinear system is locally observable at $x^{0}$. 


$$
\boldsymbol{J}=\frac{\partial \boldsymbol{Y}}{\partial \boldsymbol{x}}=\frac{\partial}{\partial \boldsymbol{x}}\left[\begin{array}{c}
h(\boldsymbol{x}, u) \\
L_{f}(h)(\boldsymbol{x}, \boldsymbol{U}) \\
L_{f}\left(h_{1}\right)(\boldsymbol{x}, \boldsymbol{U}) \\
\vdots \\
L_{f}\left(h_{n-2}\right)(\boldsymbol{x}, \boldsymbol{U})
\end{array}\right]
$$

where $\boldsymbol{Y}=\left[y, y^{\prime}, \ldots, y^{(n-1)}\right]^{\mathrm{T}}, \boldsymbol{U}=\left[u, u^{\prime}, \ldots, u^{(n-1)}\right]^{\mathrm{T}}$, and the ith derivative of the scalar output $y$ can be represented by Lie operator $L_{f}(h)$, which is the total differentiation of $h$ :

$$
\begin{aligned}
y^{(i)} & =L_{f}\left(h_{i-1}\right)(x, U) \\
& =\frac{\partial h_{i-1}}{\partial x}(x, U) f(x, u)+\frac{\partial h_{i-1}}{\partial u}(x, \boldsymbol{U}) \frac{d \boldsymbol{U}}{d t} \\
\text { e.g., } y^{\prime}=\frac{d y}{d t}=\frac{\partial h}{\partial x} f+\frac{\partial h}{\partial u} u^{\prime}= & h_{1}\left(\boldsymbol{x}, u, u^{\prime}\right)
\end{aligned}
$$

Hence, according to Theorem 1, the Jacobian matrix $J$ for the extended sub-model (4) is:

$$
J=\frac{\partial\left(y_{2}, \dot{y}_{2}\right)}{\partial\left(s o c, C_{n}\right)}=\left[\begin{array}{cc}
\frac{\mathrm{d} V_{O C}}{\mathrm{~d} s c c} & 0 \\
\Theta & \frac{\mathrm{d} V_{O C}}{\mathrm{~d} s o c} \cdot\left(\frac{\eta I_{\text {batt }}(t)}{\mathrm{C}_{\mathrm{n}}^{2}(t)}\right)
\end{array}\right]
$$

Note 1: $\dot{y}_{2}=\frac{\mathrm{d} V_{\mathrm{OC}}(s o c)}{\mathrm{d} t}=\frac{\mathrm{d} V_{\mathrm{OC}}}{\mathrm{d} s o c} \cdot \frac{\mathrm{d} s o c}{\mathrm{~d} t}=\frac{\mathrm{d} V_{\mathrm{OC}}}{\mathrm{d} s o c} \cdot\left(\frac{-\eta I_{\mathrm{bata}}(t)}{\mathrm{C}_{\mathrm{n}}(t)}\right)$

Note 2: Because of the zero in (7), $\Theta$ is just a symbol to represent the corresponding term in the $2 \times 2$ matrix, and it will have no effect on the determinant of $J$.

Therefore, the local observability condition for extended model (4) is defined by (8), where several interesting concluding remarks can be drawn.

$$
\operatorname{det}(J)=\left(\frac{\mathrm{d} V_{\mathrm{OC}}}{\mathrm{d} s o c}\right)^{2} \cdot\left(\frac{\eta I_{\text {batt }}(t)}{\mathrm{C}_{\mathrm{n}}^{2}(t)}\right) \neq 0
$$

1. The term $\left(\frac{\mathrm{d} V_{\mathrm{OC}}}{\mathrm{d} s o c}\right)^{2}$ induces the inherent weaker observability environment for the battery capacity $C_{n}$. Because $\frac{d V_{O C}}{d s o c}$ is usually smaller than one, this means its square is always smaller than itself. 2. The input $I_{\text {batt }}$ appears in the numerator of $\operatorname{det}(J)$, which means the current value will also affect the observability condition. It has been indicated in [32] that a higher absolute value of the determinant will lead to a better observability. Hence, the larger the current is, the better is the observability condition. However, the selected current level should be adequate, namely it should make a compromise between the convergence speed and the battery high-rate charging current. 3. As $C_{n}^{2}$ is in the denominator of $\operatorname{det}(J)$, then its impact cannot be ignored. A very practical problem in reality is how to choose the initial value for the estimation algorithm, namely an initial value that is larger than the reference one or smaller than the reference one, and the reason why we make that decision. With our demonstration, an initial capacity value that is smaller than the reference value is recommended when the battery capacity is estimated under the extended model framework, because this will enhance the observability condition.

To summarize, a schematic resume of the obtained necessary observability conditions for the extended battery ECM is shown in Figure 6.

Note that these observability conditions are necessary, but not sufficient for battery monitoring, because the observability of the model (3) will definitely be weaker than the observability of each sub-model. However, the proposed indirect observability analysis method can avoid applying the nonlinear observability method directly to the extended battery model, which would be tricky. 


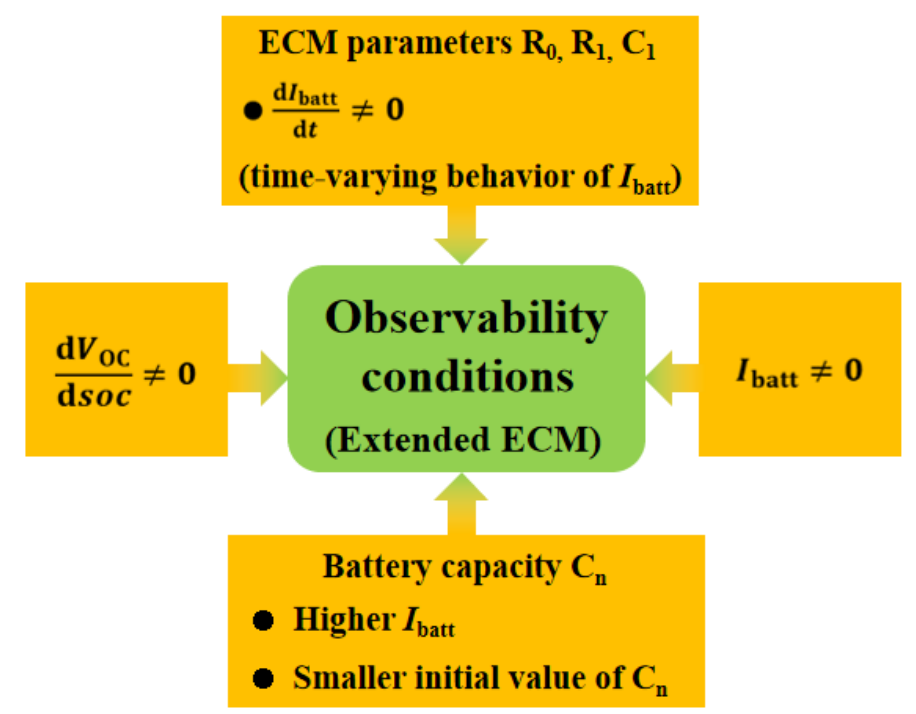

Figure 6. Schematic resume of the necessary observability conditions for extended ECM.

\section{New Cascaded Framework}

Since the battery state and parameter estimations are usually carried out in the discrete time domain inside BMS [33], the classical estimation methods will be reviewed rapidly based on the discrete time model (9) before presenting the new cascaded estimation framework. The discrete time model (9) is obtained with the zero-order hold approximation under the assumption that the current $I_{\text {batt }}$ is constant between two adjacent sampling points. $k$ is the time index.

In addition, considering the uncertainty of the modeling and data acquisition process, the stochastic model is employed instead of a deterministic one. Hence, $\omega_{1}, \omega_{2}, \omega_{2}$ are additive model errors. Using the common random walk model [19], $\omega_{4}, \omega_{5}, \omega_{6}, \omega_{7}$ are added to the slowly varying parameters. $v$ is measurement noise. The sampling period is $T$. It is assumed that all noises are additive white Gaussian (AWG).

$$
\begin{aligned}
& \left\{\begin{array}{l}
V_{1}(k)=\mathrm{e}^{\frac{-T}{\mathrm{R}_{1}(k-1) C_{1}(k-1)}} V_{1}(k-1)+\mathrm{R}_{1}(k-1) \cdot\left(1-\mathrm{e}^{\frac{-T}{\mathrm{R}_{1}(k-1) C_{1}(k-1)}}\right) \cdot I_{\text {batt }}(k-1)+\omega_{1}(k-1) \\
V_{2}(k)=\mathrm{e}^{\frac{-T}{\mathrm{R}_{2} C_{2}}} V_{2}(k-1)+\mathrm{R}_{2} \cdot\left(1-\mathrm{e}^{\frac{-T}{\mathrm{R}_{2} C_{2}}}\right) \cdot I_{\text {batt }}(k-1)+\omega_{2}(k-1) \\
s o c(k)=\operatorname{soc}(k-1)-\frac{\eta T}{\mathrm{C}_{\mathrm{n}}(k-1)} \cdot I_{\mathrm{batt}}(k-1)+\omega_{3}(k-1) \\
\mathrm{R}_{0}(k)=\mathrm{R}_{0}(k-1)+\omega_{4}(k-1) \\
\mathrm{R}_{1}(k)=\mathrm{R}_{1}(k-1)+\omega_{5}(k-1) \\
\mathrm{C}_{1}(k)=\mathrm{C}_{1}(k-1)+\omega_{6}(k-1) \\
\mathrm{C}_{\mathrm{n}}(k)=\mathrm{C}_{\mathrm{n}}(k-1)+\omega_{7}(k-1)
\end{array}\right. \\
& V_{\text {batt }}(k)=V_{\mathrm{OC}}(\text { soc })-V_{1}(k)-V_{2}(k)-\mathrm{R}_{0}(k) \cdot I_{\text {batt }}(k)+v(k)
\end{aligned}
$$

Based on (9), one way to execute the state and parameter estimation is to use a single observer, which is called the joint estimation method. Another way that resorts to two parallel observers is named as the dual estimation method, where parameter estimation can be isolated. Thus, it makes the multi-time scale estimation possible [17]. Although employing more advanced observers can improve the monitoring result based on these two classical estimation structures, the improvement is limited due to the incorrect parameter grouping. 
After obtaining the necessary observability conditions, it shows that battery usable capacity estimation has a higher demand for the observability condition. Especially, its inherent weak observability caused by the term $\left(\frac{\mathrm{d} V_{\mathrm{OC}}}{\mathrm{d} s o C}\right)^{2}$ brings the challenge. It is better to estimate the battery usable capacity separately. Hence, the new proposed cascaded framework for battery state and parameter estimation is shown in Figure 7, where $\hat{X}\left(X=S O C, R_{0}, R_{1}, C_{1}, V_{O C}, C_{n}\right)$ stands for the estimated vector. Three estimation modules can be clearly observed from this schematic representation, namely the battery capacity estimation, the battery OCV estimation, and the battery SOC and ECM parameter estimation. Besides, the aforementioned three parts are connected together by some key state variables. Details for each part will be introduced in the following.

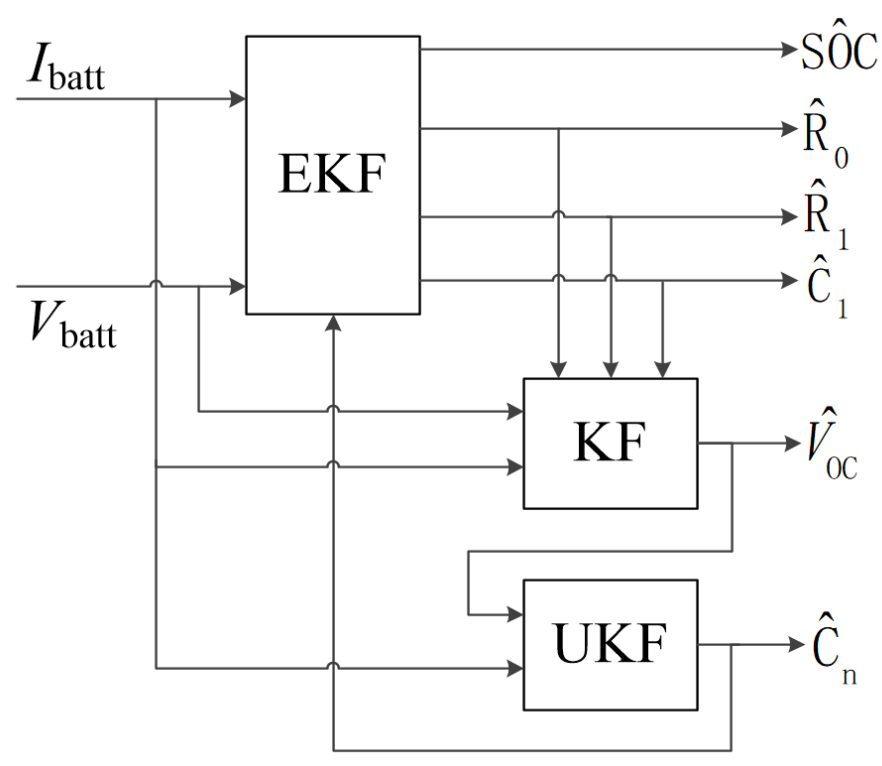

Figure 7. New proposed structure.

\subsection{Battery Usable Capacity Estimation}

A novel method to separate and estimate the battery usable capacity $C_{n}$ is introduced here, where the Coulomb counting model is reused. In addition, $V_{\mathrm{OC}}(s o c)$ is used as the output model in this module because of its inherent relationship with SOC.

Hence, after adding $C_{n}$ as an additional state, the on-line monitoring model prepared for the battery capacity is shown in (10).

$$
\begin{aligned}
& \left\{\begin{array}{l}
\operatorname{soc}(k)=\operatorname{soc}(k-1)-\frac{\eta T}{C_{\mathrm{n}}(k-1)} I_{\text {batt }}(k-1)+\gamma_{1}(k-1) \\
C_{\mathrm{n}}(k)=C_{\mathrm{n}}(k-1)+\gamma_{2}(k-1)
\end{array}\right. \\
& y_{\mathrm{O}}(k)=V_{\mathrm{OC}}(\operatorname{soc})+\delta(k)
\end{aligned}
$$

where the added noises $\gamma_{1,2}$ and $\delta$ are AWG.

Moreover, UKF, as a more powerful nonlinear observer, is selected for this two-state nonlinear model. This choice can not only guarantee the estimation performance of $C_{n}$, but also limits the increase of on-line calculation burden due to the separation.

However, the output equation $y_{\mathrm{O}}$ in (10) is the battery OCV, which can not be measured during the battery operation [34]. Hence, an OCV estimation part will be introduced later, which will be used as a measured term for the battery usable capacity estimation module. 


\subsection{Battery OCV Estimation}

The estimated battery OCV plays an important role in the new cascaded framework. As can be noticed in Figure 7, this module makes the connection between the estimated SOC and ECM parameters and the estimation of the battery usable capacity.

Thanks to the zero-time derivative condition, the estimation of the battery OCV can also be done similarly based on the extended model (11). The added noises $\varepsilon_{1 \sim 3}$ and $\psi$ are also AWG. Furthermore:

$$
\begin{aligned}
& F(k)= {\left[\begin{array}{ccc}
\mathrm{e}^{\frac{-T}{\mathrm{R}_{1}(k) C_{1}(k)}} & 0 & 0 \\
0 & \mathrm{e}^{\frac{-T}{\mathrm{R}_{2} C_{2}}} & 0 \\
0 & 0 & 1
\end{array}\right], \quad H=[-1,-1,1] } \\
& G(k)= {\left[\begin{array}{c}
\mathrm{R}_{1}(k) \cdot\left(1-\mathrm{e}^{\frac{-T}{\mathrm{R}_{1}(k) C_{1}(k)}}\right) \\
\mathrm{R}_{2} \cdot\left(1-\mathrm{e}^{\frac{-T}{\mathrm{R}_{2} C_{2}}}\right) \\
0
\end{array}\right], J(k)=-\mathrm{R}_{0}(k) } \\
& {\left[\begin{array}{c}
V_{1}(k) \\
V_{2}(k) \\
V_{\mathrm{OC}}(k)
\end{array}\right]=F(k)\left[\begin{array}{c}
V_{1}(k-1) \\
V_{2}(k-1) \\
V_{\mathrm{OC}}(k-1) \\
V_{1}(k) \\
V_{2}(k) \\
V_{\mathrm{OC}}(k)
\end{array}\right]+G(k) I_{\mathrm{batt}}(k-1)+\left[\begin{array}{l}
\varepsilon_{1}(k-1) \\
\varepsilon_{2}(k-1) \\
\varepsilon_{3}(k-1)
\end{array}\right] } \\
& V_{\text {batt }}(k)=H(k) I_{\text {batt }}(k)+\psi(k)
\end{aligned}
$$

Note that based on our former research work, although the regression-based OCV estimation algorithms can provide an independent OCV estimation, the observer-based method exhibits better OCV estimation performance from the perspective of accuracy [34]. Hence, observer-based OCV estimation is employed here in order to connect the other two parts. Besides, the estimation of the polarization voltages $V_{1}$ and $V_{2}$ cannot be simply considered as done two times, i.e., during the estimation of SOC and parameters, as well as the estimation of OCV. Because in the $k$ th step, the battery SOC and parameter estimation module use the information of the $(k-1)$ th step; while the state transfer matrix $\mathrm{F}(k)$ and the so-called control input matrix $\mathrm{G}(k)$ of $(11)$ are calculated with the latest information of the $k$ th step, namely the estimation is executed with the updated battery ECM parameters.

Obviously, the full rank characteristic of the observability matrix for this linear model can be easily verified. Therefore, the classical linear observer KF is used in this module.

\subsection{Battery SOC and ECM Parameter Estimation}

Due to the isolation of the battery usable capacity estimation, only the first six states of the state model in (9) and its measurement model will be kept and used to estimate the battery SOC and ECM parameters. Note that repetition of the Coulomb counting model is inevitable, because the measured battery terminal voltage is an integral term, which cannot be divided into different components respectively.

Besides, EKF, as one of the most used observers, is selected for this module in order to cope with the nonlinear and high-dimensional hyperspace. It should be pointed out that the battery capacity $C_{n}$ is updated by the previously mentioned part. Thus, the combination of linear and different nonlinear state tracking methods can make the new structure maintain the on-line calculation burden as small as possible. 


\section{Simulation Studies}

\subsection{Observability Conditions' Assessment}

All the battery parameters used in this paper, including the coefficients of a seventh-order polynomial OCV curve as shown in (12), were taken from [28]. They were identified through the pulsed charge and discharge tests by a pouch cell LIB. The hardware-in-the-loop validation was done in order to confirm the proposed model and parameters.

$$
\begin{gathered}
V_{\mathrm{OC}}(s o c)=a_{1} \cdot \operatorname{soc}^{7}+a_{2} \cdot \operatorname{soc}^{6}+a_{3} \cdot \operatorname{soc}^{5}+a_{4} \cdot \operatorname{soc}^{4} \ldots \\
\ldots+a_{5} \cdot \operatorname{soc}^{3}+a_{6} \cdot \operatorname{soc}^{2}+a_{7} \cdot \operatorname{soc}^{1}+a_{8}
\end{gathered}
$$

Firstly, as shown in Figure 8, three special periodic input currents $I_{\text {batt }}$ were considered. In one cycle (300 s), the LIB was discharged and recharged by a dynamic current during a small time period at the beginning; then, a constant discharge current was applied to the battery; and finally, the battery was idle until the next period. Besides, in order to verify whether the current value will affect the estimation of the battery capacity, three constant discharge values were selected, namely $10 \mathrm{~A}, 15 \mathrm{~A}$, and $20 \mathrm{~A}$.

Note that the 20 A discharge current was the default value. The EKF was initialized with the vector $x_{0}=[0,0,0.8,0.001 \Omega, 0.0035 \Omega, 17,000 \mathrm{~F}, 18 \mathrm{Ah}]$ in the simulation test if there is no further indication.

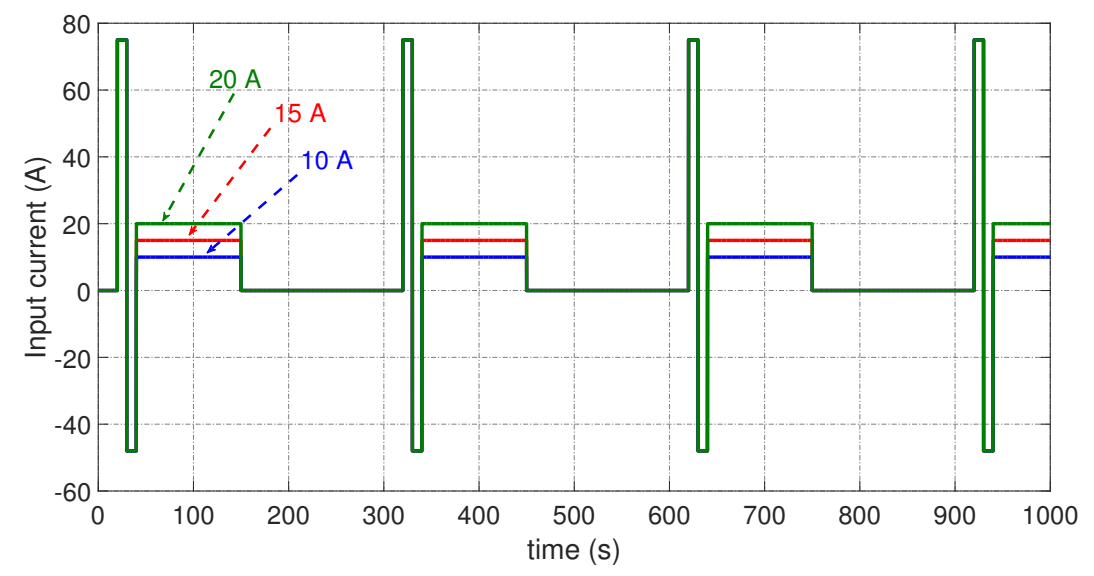

Figure 8. Three different input currents for the battery.

- $\quad \frac{\mathrm{d} I_{\text {batt }}}{\mathrm{d} t} \neq 0$ (variable battery input current)

Although there are three estimated ECM parameters, only the estimation result of $R_{0}$ will be discussed as an example. In order to verify the observability condition for the ECM parameter estimation, a simulation test was conducted in which $R_{0}$ would increase slowly in the battery model.

The result is shown in Figure 9a. The dashed line is the estimation of $R_{0}$ by EKF. Figure $9 b$ is the enlargement of the estimation result in Figure $9 a$ from $2100 \mathrm{~s}$ to $2450 \mathrm{~s}$, and Figure $9 \mathrm{c}$ is the corresponding estimation error, while Figure $9 \mathrm{~d}$ is the corresponding input current. From Figure $9 \mathrm{c}$ and Figure 9d, we can deduce that the observer estimated efficiently the resistance only when the input current was variable. Besides, the fluctuating characteristic of the estimated $R_{0}$ in Figure $9 a$ could be found evidently. This can be explained from the perspective of observability. The fluctuating characteristic of the estimated $R_{0}$ was due to the input current dynamics. 


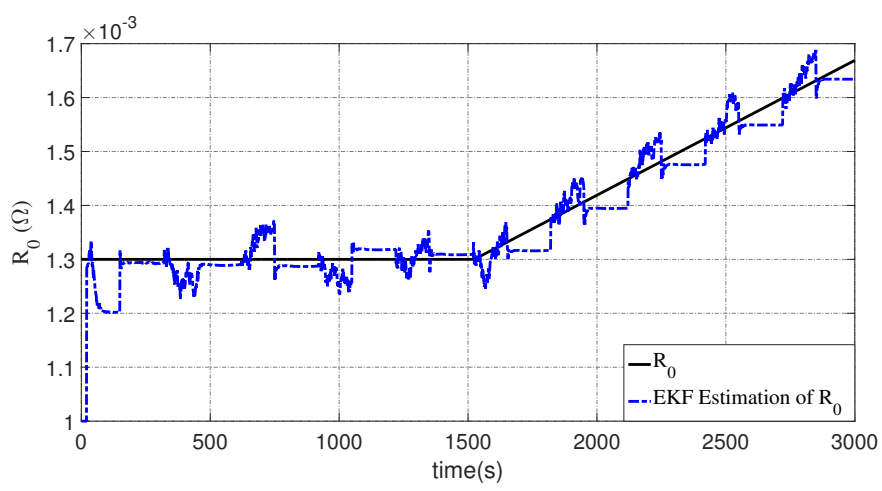

(a) Estimation test of $R_{0}$

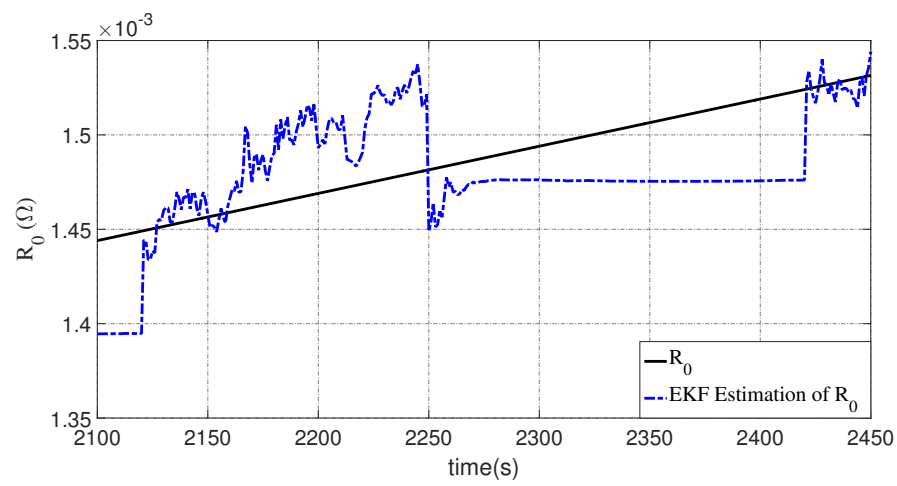

(b) Enlargement of (a) from $2100 \mathrm{~s}$ to $2450 \mathrm{~s}$

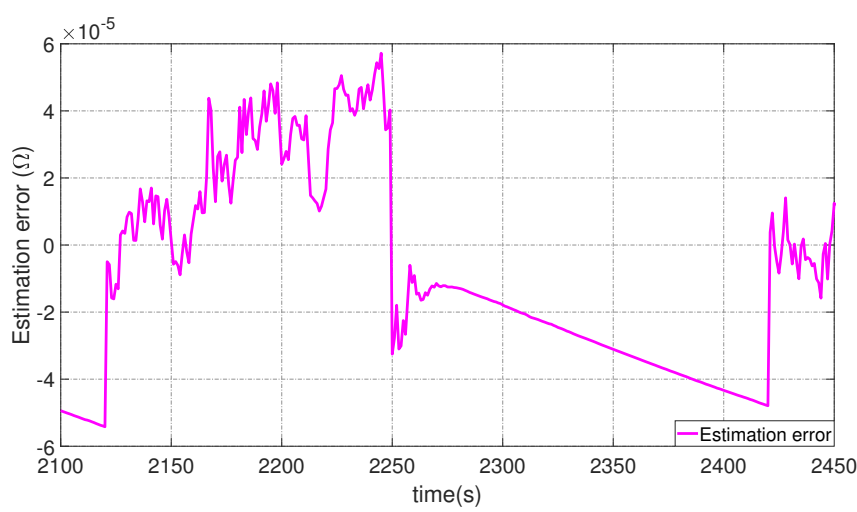

(c) Estimation error of $\mathrm{R}_{0}$ from $2100 \mathrm{~s}$ to $2450 \mathrm{~s}$

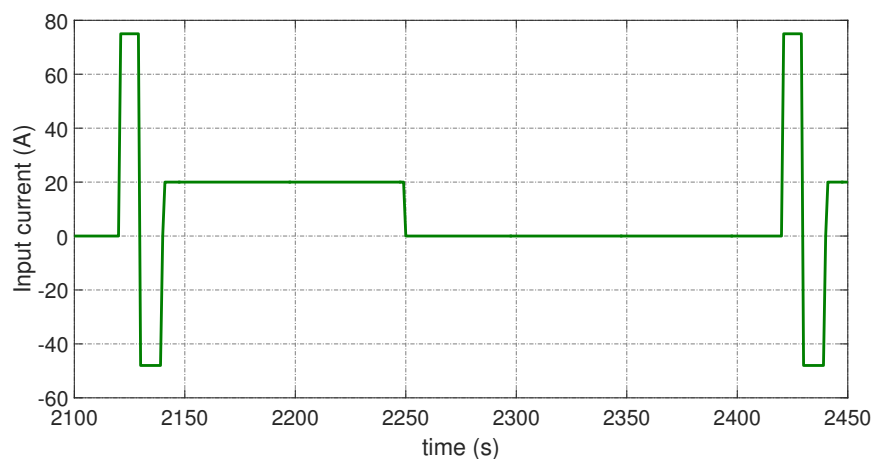

(d) $I_{\text {batt }}$ from $2100 \mathrm{~s}$ to $2450 \mathrm{~s}$

Figure 9. Verification of the time varying behavior of $I_{\text {batt }}$ on the estimation of ECM parameters. 
- $\quad$ Battery input current level

On the other hand, three previously mentioned input currents were fed to the battery ECM in order to verify whether the input current value would influence the observability condition for battery capacity $C_{n}$ estimation. Considering the effect of randomness in measurement noise, a simple quasi-Monte Carlo simulation test was conducted with 100 estimations. The estimation result based on (9) is shown in Figure 10. It confirmed that a higher value of the battery input current improved the convergence speed of the battery capacity $C_{n}$ estimation.

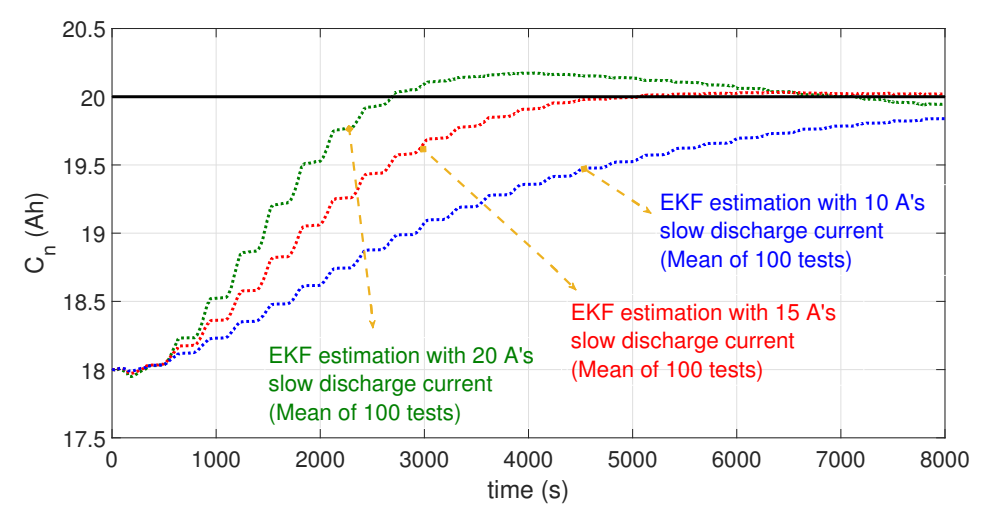

Figure 10. Battery input current level effect on battery capacity estimation.

- Initial capacity value

In order to evaluate the effect of the battery capacity initial value, the estimator was initialized with three sets of different values, namely $17 \mathrm{Ah}$ and $23 \mathrm{Ah}, 18 \mathrm{Ah}$ and $22 \mathrm{Ah}$, and $19 \mathrm{Ah}$ and 21 $\mathrm{Ah}$. To minimize the randomness effect, the estimator was executed with 100 sets of synthetic data. The estimation result in Figure 11 shows that a smaller initial value of $C_{n}$ could guarantee a rapid convergence speed for tracking the reference value. This confirmed the experimental results from [24] displayed in the Introduction of this paper.

\subsection{Evaluation of the New Estimation Structure}

The effectiveness of the new structure will be verified in the following. Comparison with estimation results from the joint estimation frameworks composed of EKF or UKF respectively will be presented.

Due to the maturity and numerous applications of KF, EKF, and UKF, the details will not be presented here. Readers can refer to $[14,17]$ to get more information. However, key information is given as follows. Firstly, the characteristics of the noises are shown in Table 1, which were set according to the existing works and several tests. For the joint estimation frameworks based on (9), the vector $\boldsymbol{x}_{\mathbf{0}}$ was used to initialize the EKF or UKF. In the new structure, the initial value of the battery OCV was set to $3.5 \mathrm{~V}$, and the other initial values were the same as in the vector $x_{0}$.

First of all, as a specific term appeared in the new structure, the estimation result of OCV is shown in Figures 12 and 13. Firstly, as is shown in Figure 12, the OCV estimation converged rapidly to the reference value and could track the dynamic change of the battery OCV. Then, from the OCV estimation error in Figure 13, KF was capable of providing an accurate estimation, which was important to connect the other two estimation modules. 

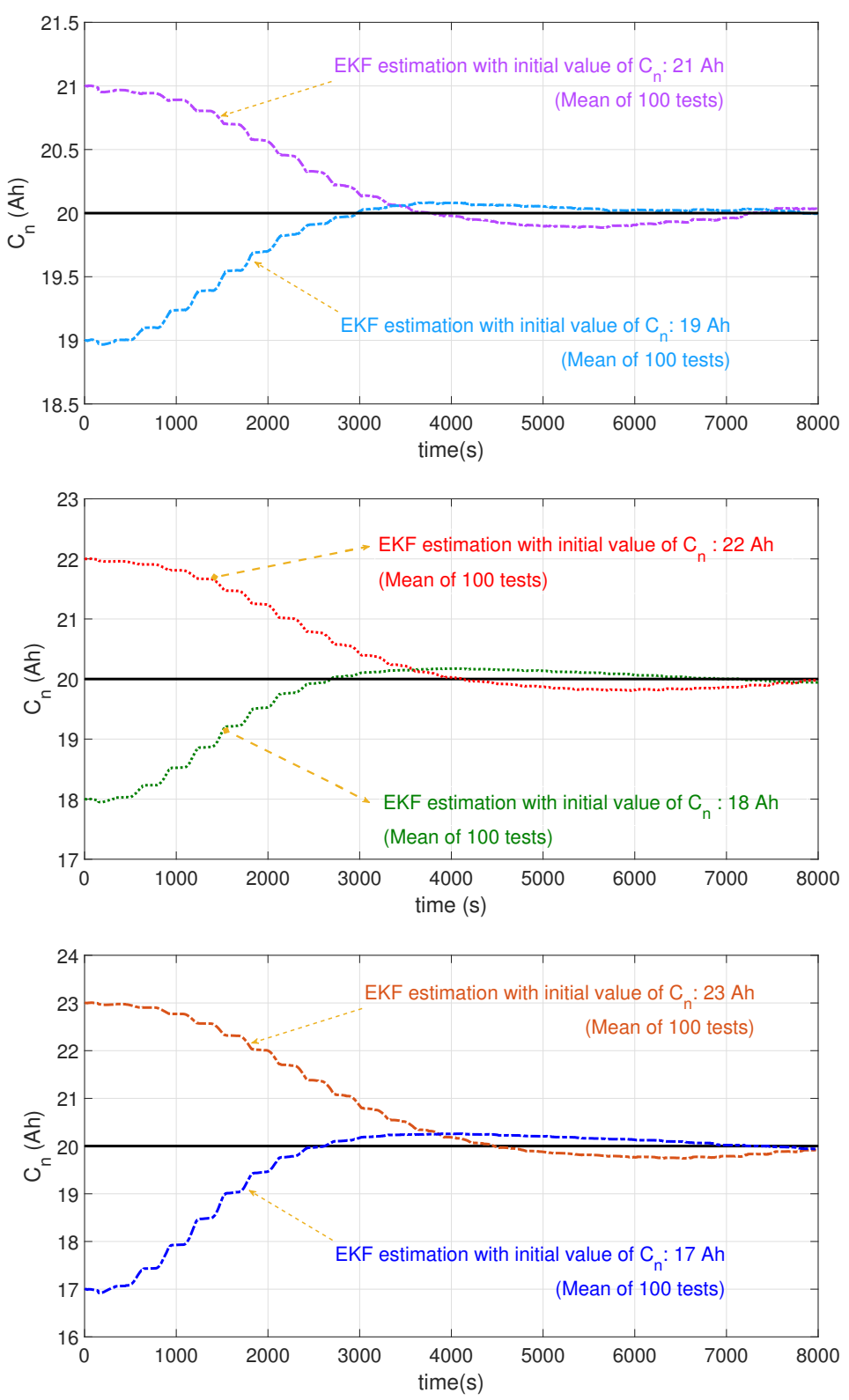

Figure 11. Initial capacity value effect on battery capacity estimation.

Table 1. Characteristics of the added noises.

\begin{tabular}{lc}
\hline$\omega_{1} \sim \mathrm{N}\left(0,1 \times 10^{-8}\right)$ & $\gamma_{1} \sim \mathrm{N}\left(0,1 \times 10^{-7}\right)$ \\
\hline$\omega_{2} \sim \mathrm{N}\left(0,1 \times 10^{-8}\right)$ & $\gamma_{1} \sim \mathrm{N}\left(0,1 \times 10^{-10}\right)$ \\
\hline$\omega_{3} \sim \mathrm{N}\left(0,1 \times 10^{-9}\right)$ & $\delta \sim \mathrm{N}\left(0,1 \times 10^{-4}\right)$ \\
\hline$\omega_{4} \sim \mathrm{N}\left(0,1 \times 10^{-12}\right)$ & $\varepsilon_{1} \sim \mathrm{N}\left(0,1 \times 10^{-8}\right)$ \\
\hline$\omega_{5} \sim \mathrm{N}\left(0,1 \times 10^{-10}\right)$ & $\varepsilon_{2} \sim \mathrm{N}\left(0,1 \times 10^{-8}\right)$ \\
\hline$\omega_{6} \sim \mathrm{N}\left(0,1 \times 10^{-10}\right)$ & $\varepsilon_{3} \sim \mathrm{N}\left(0,1 \times 10^{-6}\right)$ \\
\hline$\omega_{7} \sim \mathrm{N}\left(0,1 \times 10^{-10}\right)$ & $\psi \sim \mathrm{N}\left(0,1 \times 10^{-6}\right)$ \\
\hline$v \sim \mathrm{N}\left(0,1 \times 10^{-6}\right)$ & \\
\hline
\end{tabular}




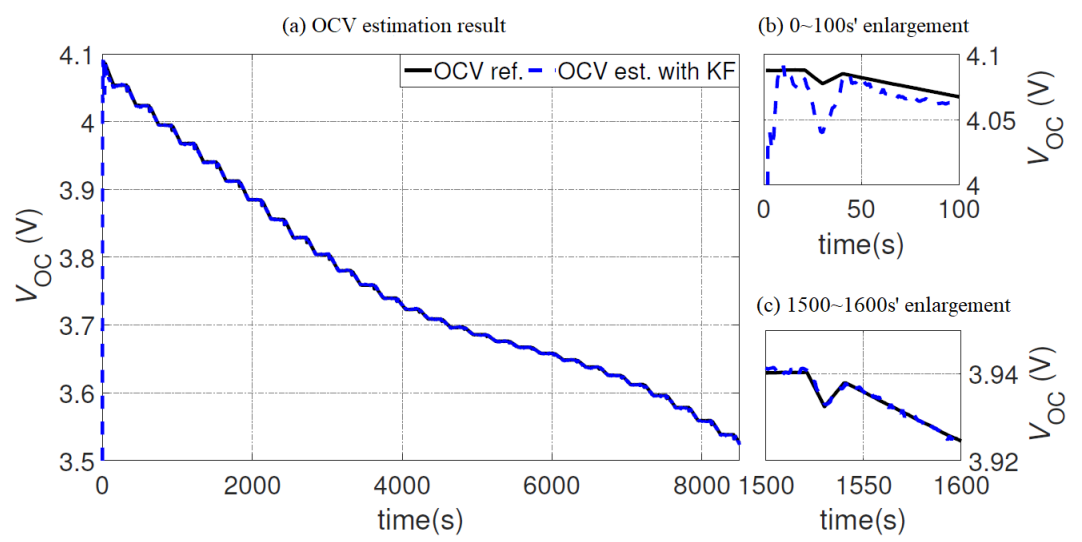

Figure 12. Estimation result of the battery OCV.

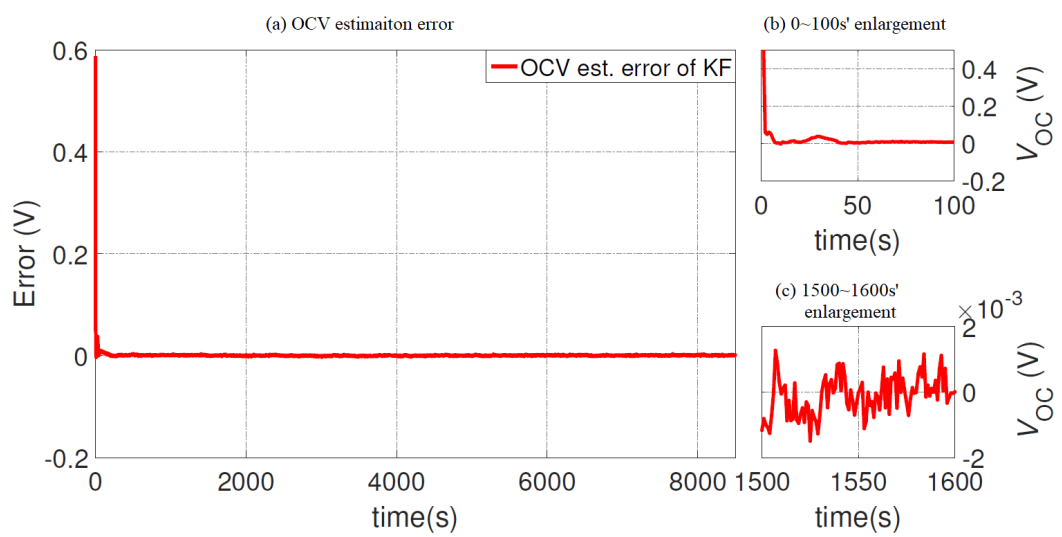

Figure 13. Estimation error of the battery OCV.

Then, the estimation results of the other parameters are shown in Figure 14. Several improvements brought by the new structure should be noticed. Firstly, as can be seen from Figure 14a,b, compared to joint EKF or joint UKF, the main improvement with the new structure came from the estimation of the battery capacity. Not only the convergence speed was more rapid, but also the estimation was fluctuating less and more accurate. Secondly, it can be observed from Figure 14c-f that the new framework improved the estimation accuracy of the RC network. Thirdly, as can be seen from Figure $14 \mathrm{i}, \mathrm{j}$, due to the improvement of the parameter estimation, the determination of the battery SOC was also improved.

Furthermore, as for the computational cost, ten simulation tests were executed for these three monitoring algorithms. As shown in Figure 14, the current cycle duration for each test was $8500 \mathrm{~s}$. The simulation was performed by using the discrete time model with a sampling period equal to 1 s, with MATLAB/Simulink R2015b, on a desktop with an Intel Core i7-6700 CPU 3.4 GHz and 8 GB RAM. The average calculation times of these ten simulation tests for each monitoring algorithm are listed in Table 2.

However, this is only a general discussion of the computation load of the newly proposed monitoring structure, because the multi-time scale can also be introduced into the new structure, which means the sampling time period for the battery capacity can be extended to a larger value according to the application. For example, the capacity estimation can even be performed quarterly or semiannually because of its slow time varying property [35]. Thus, the new structure had similar computational loads to the traditional frameworks. Although the further discussion about the multi-time scale is out of the scope of this paper, one potential advantage of the new structure should be pointed out. That is, working with the multi-time scale, the battery ECM parameters could still be estimated and updated even when the battery capacity estimation was not activated. 


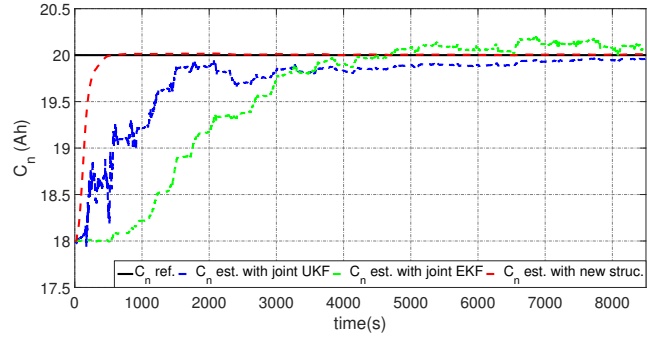

(a) Estimation result of $C_{n}$

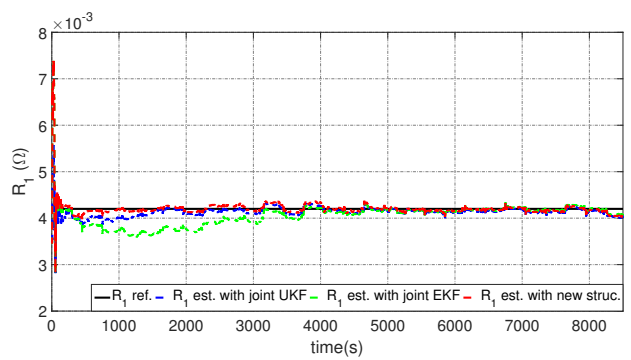

(c) Estimation result of $R_{1}$

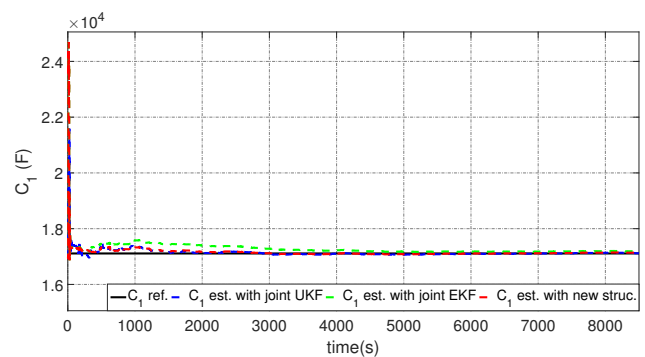

(e) Estimation result of $C_{1}$

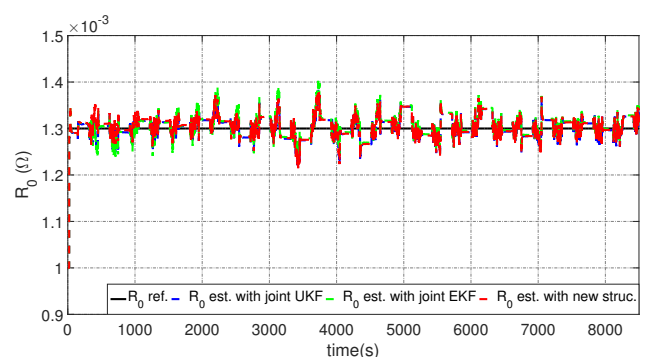

(g) Estimation result of $R_{0}$

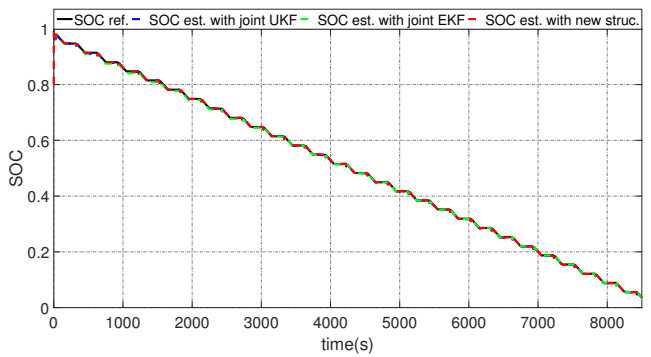

(i) Estimation result of SOC

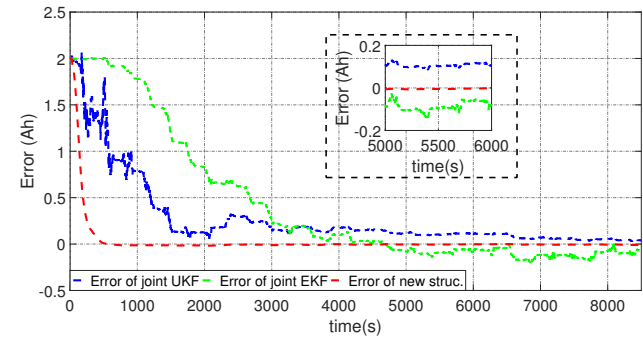

(b) Estimation error of $C_{n}$

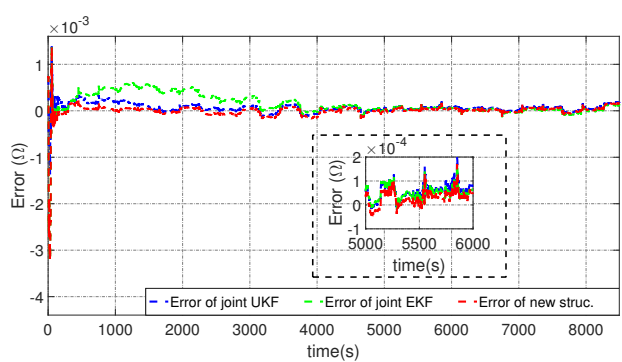

(d) Estimation error of $R_{1}$

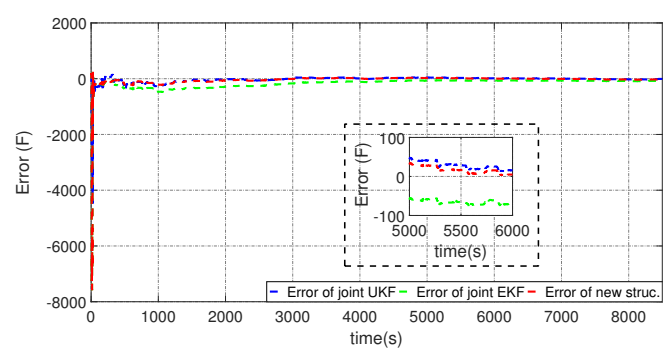

(f) Estimation error of $C_{1}$

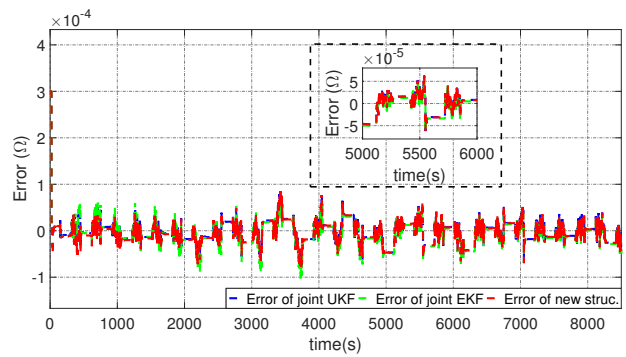

(h) Estimation error of $R_{0}$

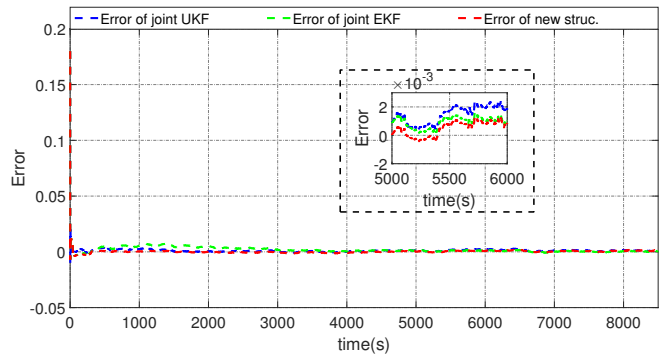

(j) Estimation error of SOC

Figure 14. Comparison of the estimation results with joint EKF, joint UKF, and the new structure. 
Table 2. Computation efficiency of the new proposed monitoring structure.

\begin{tabular}{cccc}
\hline & Joint EKF & Joint UKF & New Structure \\
\hline Average calculation time (s) & 0.264 & 3.813 & 1.986 \\
\hline
\end{tabular}

\section{Conclusions}

In this paper, a new model-based battery monitoring method was proposed. Firstly, the battery internal characteristic was fully used for the theoretical observability analysis. Namely, the linear segment characteristics of the battery OCV-SOC led to a model decomposition based on the superposition principle. Thus, the original battery ECM was decomposed into two sub-models. Each sub-model contained different battery states and parameters; hence, each of them was extended in order to be analyzed. Therefore, after the nonlinear observability analysis, the necessary observability conditions were clearly listed for the battery ECM parameters and battery capacity.

Answers to the four questions raised in the Introduction could be given as follows. Firstly, the battery capacity estimation based on the extended ECM depended on $\left(\frac{\mathrm{d} V_{\mathrm{OC}}}{\mathrm{d} s o c}\right)^{2}$, which induced the inherent weak observability. Secondly, the smaller initial value of the battery capacity would guarantee a better local observability condition, which would consequently lead to a rapid convergence speed. Thirdly, the battery state and parameter estimation based on the extended ECM depended on the battery current, namely both the current amplitude and its time varying behavior would affect the observability. Last, but not least, according to the theoretical analysis, a new cascaded framework that separated the battery ECM parameters and battery capacity estimation was proposed. The effectiveness of the analysis results and the proposed framework was validated through numerical simulations.

Author Contributions: J.M. has proposed the original idea, carried out the simulations and written the original draft paper. M.B. and D.D. have supervised the research, analyzed the results and reviewed and edited the paper. T.W. has reviewed and edited the paper. All authors have read and agreed to the published version of the manuscript.

Funding: This research was funded by the National Natural Science Foundation of China (NO. 61673260).

Conflicts of Interest: The authors declare no conflict of interest.

\section{References}

1. Tang, L.; Rizzoni, G.; Onori, S. Energy management strategy for HEVs including battey life optimization. IEEE Trans. Transp. Electrif. 2015, 1, 211-222. [CrossRef]

2. Zou, Y.; Hu, X.; Ma, H.; Li, S.E. Combined state of charge and state of health estimation over lithium-ion battery cell cycle lifespan for electric vehicles. J. Power Sources 2014, 273, 793-803. [CrossRef]

3. Ouddah, N.; Boukhnifer, M.; Raisemche, A. Two control energy management schemes for electrical hybrid vehicle. In Proceedings of the 10th International Multi-Conferences on Systems, Signals \& Devices 2013 (SSD13), Hammamet, Tunisia, 18-21 March 2013.

4. Wei, Z.; Zhao, J.; Ji, D.; Tseng, K.J. A multi-timescale estimator for battery state of charge and capacity dual estimation based on an online identified model. Appl. Energy 2017, 204, 1264-1274. [CrossRef]

5. Zou, C.; Klintberg, A.; Wei, Z.; Fridholm, B.; Wik, T.; Egardt, B. Power capacity prediction for lithium-ion batteries using economic nonlinear model predictive control. J. Power Sources 2018, 396, 580-589. [CrossRef]

6. Zhao, S.; Duncan, S.R.; Howey, D.A. Observability analysis ans state estimation of lithium-ion batteries in the presence of sensor biases. IEEE Trans. Control Syst. Technol. 2017, 25, 326-333. [CrossRef]

7. Duong, V.; Bastawrous, H.A.; Lim, K.; See, K.W.; Zhang, P.; Dou, S.X. Online state of charge and model parameters estimation of the $\mathrm{LiFePO}_{4}$ battery in electric vehicles using multiple adaptive forgetting factors recursive least-squares. J. Power Sources 2015, 296, 215-224. [CrossRef]

8. Lin, X. Theoretical analysis of battery SOC estimation errors under sensor bias and variance. IEEE Trans. Ind Electron. 2018, 65, 7138-7148. [CrossRef] 
9. Xia, B.; Lao, Z.; Zhang, R.; Tian, Y.; Chen, G.; Sun, Z.; Wang, W.; Sun, W.; Lai, Y.; Wang, M. Online parameter identification and state of charge estimation of lithium-ion batteries based on forgetting factor recursive least squares and nonlinear Kalman filter. Energies 2018, 11, 3. [CrossRef]

10. Wei, Z.; Meng, S.; Xiong, B.; Ji, D.; Tseng, K.J. Enhanced online model identification and state of charge estimation for lithium-ion battery with a FBCRLS based observer. Appl. Energy 2016, 181, 332-341. [CrossRef]

11. Wei, Z.; Zhao, J.; Xiong, R.; Dong, G.; Pou, J.; Tseng, K.J. Online estimation of power capacity with noise effect attenuation for lithium-ion battery. IEEE Trans. Ind. Electron. 2018, 66, 5724-5735. [CrossRef]

12. Wei, Z.; Dong, G.; Zhang, X.; Pou, J.; Quan, Z.; He, H. Noise-immune model identification and state of charge estimation for lithium-ion battery using bilinear parameterization. IEEE Trans. Ind. Electron. 2019. [CrossRef]

13. Åström, K.J.; Wittenmark, B. Adaptive Control, 2nd ed.; Dover Publications: New York, NY, USA, 2013.

14. Plett, G.L. Extended Kalman filtering for battery management systems of LiPB-based HEV battery packs Part 3. State and parameter estimation. J. Power Sources 2004, 134, 277-292. [CrossRef]

15. Waag, W.; Fleischer, C.; Sauer, D.U. Critical review of the methods for monitoring of lithium-ion batteries in electric and hybrid vehicles. J. Power Sources 2014, 258, 321-339. [CrossRef]

16. Plett, G.L. Dual and joint EKF for simultaneous SOC and SOH estimation. In Proceedings of the 21st Electric Vehicle Symposium, Berlin, Germany, 2-6 April 2005.

17. Plett, G.L. Sigma-point Kalman filtering for battery management systems of LiPB-based HEV battery packs Part 2: Simultaneous state and parameter estimation. J. Power Sources 2006, 161, 1369-1384. [CrossRef]

18. He, H.; Xiong, R.; Guo, H. Online estimation of model parameters and state-of-charge of $\mathrm{LiFePO}_{4}$ batteries in electric vehicles. Appl. Energy 2012, 89, 413-420. [CrossRef]

19. Zhao, L.; Liu, Z.; Ji, G. Lithium-ion battery state of charge estimation with model parameters adaption using $H_{\infty}$ extended Kalman filter. Control Eng. Pract. 2018, 81, 114-128. [CrossRef]

20. Kim, I.-S. A technique for estimating the state of health of lithium batteries through a dual-sliding-mode observer. IEEE Trans. Power Electron. 2010, 25, 1013-1022. [CrossRef]

21. Chen, C.; Xiong, R.; Shen, W. A lithium-ion battery-in-the-loop approach to test and validate multiscale dual $\mathrm{H}$ infinity filters for state-of-charge and capacity estimation. IEEE Trans. Power Electron. 2018, 33, 332-342. [CrossRef]

22. Xiong, R.; Zhang, Y.; He, H.; Zhou, X.; Pecht, M.G. A double-scale, particle-filtering, energy state prediction algorithm for lithium-ion batteries. IEEE Trans. Ind. Electron. 2018, 65, 1526-1538. [CrossRef]

23. Yu, Q.; Xiong, R.; Lin, C.; Shen, W.; Deng, J. Lithium-ion battery parameters and state-of-charge joint estimation based on H-infinity and unscented Kalman filters. IEEE Trans. Veh. Technol. 2017, 66, 8693-8701. [CrossRef]

24. Hu, C.; Youn, B.D.; Chung, J. A multiscale framework with extended Kalman filter for lithium-ion battery SOC and capacity estimation. Appl. Energy 2012, 92, 694-704. [CrossRef]

25. Lin, X.; Perez, H.E.; Mohan, S.; Siegel, J.B.; Stefanopoulou, A.G.; Ding, Y.; Castanier, M.P. A lumped-parameter electro-thermal model for cylindrical batteries. J. Power Sources 2014, 257, 1-11. [CrossRef]

26. Einhorn, M.; Conte, F.V.; Kral, C.; Fleig, J. Comparison, selection, and parameterization of electrical battery models for automotive applications. IEEE Trans. Power Electron. 2013, 28, 1429-1437. [CrossRef]

27. Yu, Q.; Xiong, R.; Wang, L.; Lin, C. A comparative study on open circuit voltage models for lithium-ion batteries. Chin. J. Mech. Eng. 2018, 31, 1-8. [CrossRef]

28. Chen, Z.; Fu, Y.; Mi, C.C. State of charge estimation of lithium-ion batteries in electric drive vehicles using exended Kalman filtering. IEEE Trans. Veh. Technol. 2013, 62, 1020-1030. [CrossRef]

29. Chiang, Y.H.; Sean, W.Y.; Ke, J.C. Online estimation of internal resistance and open-circuit voltage of lithium-ion batteries in electric vehicles. J. Power Sources 2011, 196, 3921-3932. [CrossRef]

30. Ogata, K. Control System Design in State Space. In Modern Control Engineering, 5th ed.; Prentice Hall: Upper Saddle River, NJ, USA, 2009; pp. 751-792.

31. Shi, Z.; Auger, F.; Schaeffer, E.; Guillemet, P.; Loron, L. Interconnected observers for online supercapacitor ageing monitoring. In Proceedings of the IECON 2013-39th Annual Conference of the IEEE Industrial Electronics Society, Vienna, Austria, 10-13 November 2013.

32. Hua, Y.; Cordoba-Arenas, A.; Warner, N.; Rizzoni, G. A multi time-scale state-of-charge and state-of-health estimation framework using nonlinear predictive filter for lithium-ion battery pack with passive balance control. J. Power Sources 2015, 280, 293-312. [CrossRef] 
33. Hauser, A.; Kuhn, R. High-voltage battery management systems (BMS) for electric vehicles. In Advances in Battery Technologies for Electric Vehicles; Woodhead Publishing: Sawston, UK, 2015; pp. 265-282,

34. Meng, J.; Boukhnifer, M.; Diallo, D. A comparative study of open-circuit-voltage estimation algorithms for lithium-ion batteries in battery management systems. In Proceedings of the 6th IEEE Internal conference on Control, Decision and Information Technologies, Paris, France, 23-26 April 2019.

35. Shen, P.; Ouyang, M.; Lu, L.; Li, J.; Feng, X. The co-estimation of state of charge, state of health, and state of function for lithium-ion batteries in electric vehicles. IEEE Trans. Veh. Technol. 2018, 67, 92-103. [CrossRef]

2020 by the authors. Licensee MDPI, Basel, Switzerland. This article is an open access article distributed under the terms and conditions of the Creative Commons Attribution (CC BY) license (http://creativecommons.org/licenses/by/4.0/). 